\title{
Adenosine Triphosphate Neutralizes Pneumolysin-induced \\ Neutrophil Activation
}

Fabian Cuypers, ${ }^{1}$ Björn Klabunde, ${ }^{1, \#}$ Manuela Gesell Salazar, ${ }^{2}$ Surabhi Surabhi, ${ }^{1}$ Kolnfekt Study Group, Sebastian B. Skorka, ${ }^{1}$ Gerhard Burchhardt, ${ }^{1}$ Stephan Michalik, ${ }^{2}$ Thomas Thiele, ${ }^{3}$ Manfred Rohde, ${ }^{4}$ Uwe Völker, ${ }^{2}$ Sven Hammerschmidt, ${ }^{1}$ Nikolai Siemens ${ }^{1}$

${ }^{1}$ Department of Molecular Genetics and Infection Biology, Interfaculty Institute for Genetics and Functional Genomics, Center for Functional Genomics of Microbes, University of Greifswald, Greifswald, Germany, ${ }^{2}$ Department of Functional Genomics, Interfaculty Institute for Genetics and Functional Genomics, Center for Functional Genomics of Microbes, University Medicine Greifswald, Greifswald, Germany, ${ }^{3}$ Institute for Immunology and Transfusion Medicine, University Medicine Greifswald, Greifswald, Germany ${ }^{4}$ Central Facility for Microscopy, Helmholtz Centre for Infection Research, HZI, Braunschweig, Germany, \#current address: Institute for Lung Research, Philipps-University Marburg, Marburg, Germany

\section{Correspondence:}

Nikolai Siemens, Department of Molecular Genetics and Infection Biology, Center for Functional Genomics of Microbes, University of Greifswald, Felix-Hausdorff-Straße 8, D17489 Greifswald, Germany, phone: +49 (0)3834 4205711 (nikolai.siemens@unigreifswald.de). 
summary: Granule effector proteins of neutrophils play a crucial role in pneumococcal clearance. Uncontrolled neutrophil degranulation can result in tissue pathology. Pneumolysin is a major pneumococcal cytoplasmic trigger of neutrophil degranulation. Extracellular ATP binds pneumolysin and neutralizes excessive pneumolysin-mediated neutrophil activation. 


\section{ABSTRACT}

Background. In tissue infections, adenosine triphosphate (ATP) is released into extracellular space and contributes to purinergic chemotaxis. Neutrophils are important players in bacterial clearance and recruited to the site of tissue infections. Pneumococcal infections can lead to uncontrolled hyper-inflammation of the tissue along with substantial tissue damage through excessive neutrophil activation and uncontrolled granule release. We aimed to investigate the role of ATP in neutrophil response to pneumococcal infections.

Methods. Primary human neutrophils were exposed to the pneumococcal strain TIGR4 and its pneumolysin deficient mutant or directly to different concentrations of recombinant pneumolysin. Neutrophil activation was assessed by measurement of secreted azurophilic granule protein resistin and profiling of the secretome, using mass spectrometry.

Results. Pneumococci are potent inducers of neutrophil degranulation. Pneumolysin was identified as a major trigger of neutrophil activation. This process is partially lysis independent and inhibited by ATP. Pneumolysin and ATP interact with each other in the extracellular space leading to reduced neutrophil activation. Proteome analyses of the neutrophil secretome confirmed that ATP inhibits pneumolysin-dependent neutrophil activation.

Conclusions. Our findings suggest that despite its cytolytic activity, pneumolysin serves as a potent neutrophil activating factor. Extracellular ATP mitigates pneumolysin induced neutrophil activation.

Keywords. neutrophils, pneumolysin, ATP, Streptococcus pneumoniae, degranulation 


\section{BACKGROUND}

Neutrophil migration to the site of infection is a crucial step in host defense. During an infection and subsequent hyper-inflammation, multiple cell types release nucleotides including adenosine triphosphate (ATP) [1]. ATP belongs to the divers group of dangerassociated molecular patterns (DAMPs) and its release from intracellular into extracellular space is associated with devastating conditions such as sepsis and shock [2]. In the course of tissue infections, extracellular ATP levels can rise up to $\mathrm{mM}$ ranges [3, 4]. Neutrophils transmigrate towards a chemotactic gradient of inflammatory mediators. The presence of extracellular ATP stimulates purinergic signaling through P2 receptors and enhances neutrophil migration, referred to as purinergic chemotaxis [5]. It was shown that P2 receptor deficient mice show increased susceptibility to Pseudomonas aeruginosa lung infections [6], which suggests that extracellular ATP and P2 receptors potentially exert a protective role against infection of the lungs.

Once neutrophils have reached the infection site, three distinct mechanisms to fight an infection occur: phagocytosis, degranulation, and formation of neutrophil extracellular traps (NETs) [7-9]. Critical components of all these processes are granule effector molecules, including proteolytic enzymes and antimicrobial peptides and proteins [10]. The release of granule components, a process called degranulation, is tightly controlled. At the site of infection, receptor recognition induces cytoskeletal remodeling, which leads to mobilization of the granules to the outer membrane. Once the neutrophil membrane is reached, granules fuse with the neutrophil membrane, which in turn results in exocytosis of granular components [11]. Although neutrophils and neutrophil-derived components are crucial for clearance of the pathogens, they also cause substantial tissue damage $[8,12,13]$. In mouse models of pneumococcal lung infections, early neutrophil recruitment was associated with a decrease of bacterial burden in the lungs [14]. However, persistent neutrophil influx was linked to disease severity at later stages of infection [14]. 
Streptococcus pneumoniae (pneumococcus) is a Gram-positive bacterium that frequently colonizes asymptomatically the upper respiratory tract of healthy individuals [15]. However, imbalances in the immune system can lead to migration of pneumococci resulting in severe life-threatening diseases such as pneumonia, meningitis, and sepsis [16]. It is widely accepted that pneumococci are the leading cause of community-acquired pneumonia [17]. Pneumococci are equipped with an arsenal of virulence factors responsible for disease manifestation and progression [18, 19]. Among them, pneumolysin (Ply) is one of the major pneumococcal virulence factors [20]. Ply is a cytolysin, which is virtually expressed by all pneumococci [19]. It oligomerizes as rings on cholesterol-rich lipid membranes and triggers formation of membrane pores [21]. Recently it was discovered that Ply interacts with mannose receptor $C$ type 1 (MRC-1/CD206) on human dendritic cells (DCs) and mouse alveolar macrophages [22]. This interaction allows pneumococci to dampen proinflammatory responses and to establish residency in the airways [22]. In contrast to other immune cells, neutrophil activation and degranulation in response to Ply is relatively unexplored. It was shown that Ply induces neutrophil activation, leading to a release of neutrophil elastase (NE) [23]. Subsequently, NE induces detachment of alveolar epithelial cells [23]. The authors suggested that $P 2 X_{7}$ receptor $\left(P 2 X_{7} R\right)$, which is an ionotropic ATP receptor, is involved in Ply-mediated neutrophil activation [23]. Furthermore, it was shown that Ply promotes formation of pro-thrombotic neutrophil-platelet aggregates [24]. Ullah and colleagues have demonstrated that although pneumococcus is a potent stimulator of NET formation, Ply itself is not required for this process [25].

Here, we investigated the degree and specificity of Ply-mediated activation of human primary neutrophils and the role of ATP-P2X ${ }_{7}$ R-axis in this process. We show that even at sub-lytic concentrations Ply is a potent inducer of neutrophil degranulation. Furthermore, we demonstrate that the activating properties of Ply are neutralized by extracellular ATP. 


\section{METHODS}

\section{Ethics Statement}

Blood samples from healthy volunteers were used in this study. Donors were individuals well acquainted with the research conducted and written informed consent was obtained. The ethical research committee at the University Medicine Greifswald approved the study (BB 006/18). All experiments were carried out in accordance with the approved guidelines.

\section{Bacterial Strains and Culture Conditions}

S. pneumoniae TIGR4 and its isogenic mutant $\Delta p l y$ were cultured to exponential growth phase $\left(\mathrm{OD}_{600}, 0.35\right.$ to 0.4$)$ in Todd-Hewitt broth supplemented with $0.5 \%(\mathrm{w} / \mathrm{v})$ yeast extract (THY; Roth).

For construction of pASK-IBA5_Ply expression vector, genomic DNA from S. pneumoniae TIGR4 wild-type strain was purified. Ply was amplified with Phusion polymerase (NEB) by using the following primer sets: forward, 5'-CGGGATCCGCAAATAAAGCAGTAAATGAC-3' and reverse, 5'-GCGGTACCTAGTCATTTTCTACCTGAG-3'. The amplified fragments were digested with BamHI and Kpnl and ligated into the vector pASK-IBA2 (IBA). For expression, Escherichia coli DH5a containing the PASK-IBA5_Ply expression vector was cultured in LB medium at $37^{\circ} \mathrm{C}$ with shaking. At $\mathrm{OD}_{550} 0.6-0.8$, expression was induced with $200 \mathrm{ng} / \mathrm{ml}$ anhydrotetracycline, the cells were incubated for $3 \mathrm{~h}$ at room temperature with shaking, and processed as detailed previously [13]. The recombinant Ply appeared as a single band of the correct molecular weight as determined by SDS-PAGE and silver staining (Supplementary Figure 2A). 


\section{Pneumococcal Survival in Complex Media}

The survival assay was performed as previously described [26]. Briefly, a $20 \mu$ l aliquot of the suspension containing $5 \times 10^{4}$ wild-type and $\Delta$ ply pneumococci was inoculated into $480 \mu \mathrm{l}$ of heparinized human blood, plasma or THY medium. After $2 \mathrm{~h}$ of incubation at $37^{\circ} \mathrm{C}$ with rotation, the CFU were determined by plating serial dilutions on blood agar plates and compared to the initial inoculum.

\section{Isolation and Stimulation of Primary Human Neutrophils}

Human neutrophils were isolated from healthy donors using a density gradient centrifugation on Polymorphprep (Axis Shield) [12]. After isolation, neutrophils were either suspended in Hank's balanced salt solution (HBSS; Life Technologies) supplemented with autologous plasma $(5 \%[\mathrm{v} / \mathrm{v}])$ or RPMl 1640 medium supplemented with $10 \mathrm{mM} \mathrm{L-glutamine,} 25 \mathrm{mmol} / \mathrm{L}$ HEPES (all HyClone) and 5\% FCS. $5 \times 10^{5}$ neutrophils were infected with bacteria (MOI 10) in RPMI medium or stimulated with different concentrations of recombinant Ply, ATP (Invivogen), or dilutions of bacterial lysates for $2 \mathrm{~h}$. To inhibit $\mathrm{P} 2 \mathrm{X}_{7} \mathrm{R}$, neutrophils were treated with AZ10606120 (R\&D Systems) for $30 \mathrm{~min}$ prior to stimulations. Neutrophil supernatants were collected and stored for further analyses at $-80^{\circ} \mathrm{C}$.

Cytotoxicity was determined by measurement of the LDH activity via CytoTox 96 Non-Radio Kit (Promega) according to manufacturer's guidelines.

For immune analysis, neutrophils were fixed in $2 \%(\mathrm{v} / \mathrm{v})$ formaldehyde, and immunostained as previously described $[12,27]$. The following reagents were used: rabbit anti-Ply antibody (in-house production by the laboratory of Sven Hammerschmidt), Nuclear-ID Red DNA stain (Enzo Life Science). Specific Ply-staining was detected by Alexa 488-conjugated goat antirabbit IgG (Molecular Probes). The staining was visualized using an Axio Observer Z1 microscope (Zeiss) or FACSAria III (BD). 


\section{Human Resistin Enzyme-linked Immunosorbent Assay}

Resistin concentrations in supernatants of neutrophils were measured by an ELISA (BioVendor) according to the manufacturer's instructions.

\section{Microscale Thermophoresis (MST)}

MST analyses were performed as previously described [28]. Briefly, recombinant Ply was NT-647 labeled according to manufacturer's guidelines (NanoTemper Technologies). The concentration of NT-647 labeled Ply was kept constant $(2.6 \mu \mathrm{M})$, while the concentrations of non-labeled ATP, dATP, and ADP varied (up-to $100 \mathrm{mM}$ ). The assay was performed in MST buffer (50 mM Tris-HCl pH 7.8, $150 \mathrm{mM} \mathrm{NaCl}, 10 \mathrm{mM} \mathrm{MgCl}_{2}$ ) containing $0.05 \%$ Tween. The samples were loaded into Monolith NT.115 capillaries and the MST was performed using a Monolith NT.115 machine and MO control software (NanoTemper Technologies). The resulting plot was analyzed via MO Affinity Analysis software (NanoTemper Technologies) by fitting a 1:1 binding model $\left(K_{d}\right.$ model).

\section{Field Emission Scanning Electron Microscopy}

Field emission scanning electron microscopy (FESEM) analysis was performed as previously described [29]. Detailed protocol is summarized in the supplementary file.

\section{Statistical Analysis}

If not otherwise indicated, statistical significance of differences was determined using Kruskal Wallis test with Dunn's post-test. Statistics were performed using GraphPad Prism version 7 (GraphPad software). A p-value less than 0.05 was considered significant. 


\section{Protein Extraction, Digestion for Mass Spectrometry (MS), Identification,}

\section{Quantification, and Data Analysis}

For proteomic analyses $500 \mu \mathrm{l}$ neutrophil supernatant were reduced with dithiothreitol $\left(2.5 \mathrm{mM}, 60 \mathrm{~min}, 60^{\circ} \mathrm{C}\right)$ and alkylated with iodo acetamid $\left(10 \mathrm{mM}, 30 \mathrm{~min}, 37^{\circ} \mathrm{C}\right.$, dark). Proteins were enriched using $2 \mu \mathrm{l}$ of SP3 beads (hydrophobic: Sera-Mag Speedbeads carboxylate-modified particles [Thermo Fisher Scientific]; hydrophilic: Speedbead magnetic carboxylate-modified particles [GE Healthcare]). Beads were added to each sample and solutions were adjusted to $70 \%(\mathrm{v} / \mathrm{v})$ with acetonitrile:water. For the on-bead protein digestion with trypsin and for peptide purification an adapted SP3 protocol was used as described previously [30]. Peptides were suspended in $2 \%$ (v/v) dimethyl sulfoxide and mixed with equal amounts of $4 \%(\mathrm{v} / \mathrm{v})$ acetonitrile. Tryptic peptide solutions were analyzed by LC-MS/MS on a reverse phase HPLC chromatography (UltiMate 3000 nano-LC system, Thermo Fisher Scientific) coupled to an $Q$ Exactive ${ }^{\mathrm{TM}}$ HF mass spectrometer in a data independent acquisition mode. To generate a specific ion library, pools of the supernatants of different donors of each group were analyzed in data dependent acquisition mode. Instrument setup and methods for data dependent and independent acquisition mode are displayed in Supplementary Tables S1-S3.

The ion library was constructed with Spectronaut version 13 (Biognosys AG) based on a database search against a Uniprot/Swissprot database limited to human entries (version 04/2019), using trypsin/P digest rule with a maximum number of two missed cleavages, carbamidomethylation as static, methionine oxidation and acetylation (protein terminus) as variable modifications. The analyses of DIA data were performed using Spectronaut version 13 (Biognosys AG). Settings are specified in Supplementary Table S4.

Further DIA-MS data analysis was performed using $R$ (R Version 3.6.1) using the tidyverse package (version 1.2.1) according to the following steps: (i) median normalization of the Spectronaut raw data over MS2 total peak area intensities (EG.TotalQuantity) for all ions ( $q$ value $<0.01$ ), (ii) replacement of zero intensity values by half-minimal intensity value from the 
whole dataset, (iii) calculation of the sum over ions per sample and peptide to generate peptide intensity data, and (iv) generation of $\mathrm{Hi} 3$ protein-intensity data. For proteins identified with at least two peptides the ROPECA statistical analysis [31] on peptide level was performed using the median for the aggregation of the signal ratios to the signal log2 ratio on protein level [32]. The principle component analysis of the scaled data was carried out using the FactoMineR package (version: 1.41). Plots were generated using the FactoMineR package (version: 1.41), factoextra package (version: 1.0.5), tidyverse package (version: 1.2.1). Functional enrichment analyses were carried with the $g$ :Profiler tool, version e99_eg46_p14_f929183, using the g:SCS (Set Counts and Sizes) correction method [33]. 


\section{RESULTS}

\section{S. pneumoniae Activates Neutrophils}

First, we assessed the role of Ply in bacterial survival in complex medium and ex vivo in human plasma and blood. TIGR4 wt and $\Delta p / y$ pneumococci were incubated in THY, freshly collected human blood or plasma for $2 \mathrm{~h}$. Viable bacteria were determined by plating serial dilutions. Both strains survived in THY and human plasma (Figures $1 \mathrm{~A}$ and $1 \mathrm{~B}$ ). However, the bacteria were efficiently killed in blood (Figure 1C) indicating that cellular blood components are responsible for killing of the pathogen. Since neutrophils are the major leukocyte component of the blood and the first responders, we assessed neutrophil activation. The analyses of resistin as an activation marker revealed that both strains efficiently activate neutrophils (Figure 1D). No differences in bacterial survival and neutrophil activation between TIGR4 wt and the isogenic $\Delta p l y$ mutant were observed.

\section{Pneumolysin is a Potent Trigger of Neutrophil Activation}

To exclude potential additive effects of activation due to other cellular and acellular compartments of the whole blood, purified human neutrophils were incubated with the bacteria or bacterial lysates and cytotoxicity towards the cells and degranulation were assessed. When challenged with bacteria, only minor cytotoxic effects towards neutrophils were noted (Figures 2A). Both, TIGR4 wt and $\Delta p l y$ mutant induced degranulation of neutrophils to an equal extent (Figure 2B). Next, neutrophils were stimulated with the cytoplasmic fraction of bacterial lysates. Although TIGR4 wt lysates were highly hemolytic (Supplementary Figure S1), only minor cytolytic effects towards neutrophils were noted (Figure $2 \mathrm{C}$ ). However, neutrophils responded by releasing high amounts of resistin (Figure 2D). Lysates of the $\Delta p l y$ mutant strain did not induce cytotoxicity or neutrophil activation (Figures $2 \mathrm{C}$ and $2 \mathrm{D}$ ). This result suggested that Ply is one of the major pneumococcal cytoplasmic components responsible for activation of neutrophils and this effect is not fully lysis dependent. 


\section{Exogenous ATP Neutralizes Ply-induced Neutrophil Lysis and Activation}

It was suggested that $\mathrm{P} 2 \mathrm{X}_{7} \mathrm{R}$, which serves as an ATP receptor, is involved in Ply-induced neutrophil activation [23]. Therefore, we have assessed direct effects of active purified Ply (Supplementary Figure S2A-C) and exogenous ATP in neutrophil activation. First, neutrophils were stimulated with different concentrations of Ply with and without supplementation of autologous plasma. Concentration dependent lysis of neutrophils was observed (Figure 3A). Based on cytotoxicity tests with plasma supplementation, $0.3125 \mu \mathrm{g}$ Ply were defined as sub-lytic amount of the toxin. In contrast to the results of neutrophil viability post Ply stimulations, even sub-lytic concentrations of Ply induced neutrophil degranulation (Figure 3B). No significant differences between sub-lytic and lytic amounts of Ply in neutrophil activation were observed (Figure 3B). The presence of Ply on neutrophils was further confirmed via immuno-fluorescence microscopy. Ply was readily detectable on neutrophils incubated with $0.3125 \mu \mathrm{g}$ Ply (Figure 3C). However, autologous plasma supplementation reduced neutrophil activation due to the presence of anti-Ply antibodies (Supplementary Figure S2D). To avoid antibody-mediated inhibitory effects, all following experiments were performed without plasma supplementation.

Next, neutrophils were stimulated with sub-lytic $\left(0.3125 \mu \mathrm{g} \mathrm{ml}^{-1}\right)$ and lytic $\left(2.5 \mu \mathrm{g} \mathrm{ml}^{-1}\right)$ Ply concentrations in the presence or absence of $P 2 X_{7} R$ agonist ATP and/or specific pharmacological P2X ${ }_{7}$ R-inhibitor AZ10606120 (Figure 4, Supplementary Figure S3). Increasing concentrations of ATP reduced Ply-mediated neutrophil lysis (Supplementary Figure S3) and degranulation (Figure 4A), while AZ10606120 had no significant effect in any of the experiments. $3 \mathrm{mM}$ ATP almost completely abolished or significantly reduced excessive neutrophil degranulation when sub-lytic or lytic concentrations of Ply were used, respectively (Figure $4 \mathrm{~A})$. 


\section{Secretome Analyses of Neutrophils}

To verify the observed results on the global level, secretome composition of neutrophils was quantitatively profiled by mass spectromentry. The presence of $3 \mathrm{mM}$ ATP substantially reduced Ply-mediated degranulation (Figure 4B). Significantly less peptides/proteins were detected in samples which were subjected to both, Ply and ATP as compared to single Ply stimulations (Figure 4B, Supplementary Table S1). Furthermore, principal component analysis (PCA) showed that both groups of Ply stimulations, sub-lytic and lytic concentrations, cluster together, suggesting that a sub-lytic amount of Ply is sufficient to activate neutrophils (Figure 4C). The analyses further confirmed that neutrophil stimulation with sub-lytic concentrations of Ply leads to a release of granule content which is almost equivalent to the content found in lysis (Figure 4B, Supplementary Tables S5 and S6). Only 54 proteins/peptides showed altered abundance in neutrophil supernatants after different Ply stimulations (Figure 4D, Supplementary Tables S5 and S6). In addition, comparison of only Ply and respective additive ATP stimulations of neutrophils revealed substantial reduction of protein amounts in supernatants if ATP was used (Figures 5A and 5B, Supplementary Table S6). Only seven proteins, which belong to the cytosolic fraction were found in higher abundance in neutrophil supernatants post Ply/ATP stimulations (Figure 5A). Pathway analyses by $g$ :Profiler confirmed that the use of ATP inhibits major neutrophil defense mechanisms, including activation, degranulation, and immune responses (Figure 5C).

\section{ATP Binds to Ply and Subsequently Reduces its Binding to Neutrophils}

Since our experiments suggest that $\mathrm{P} 2 \mathrm{X}_{7} \mathrm{R}$ does not seem to be involved in Ply-mediated neutrophil activation, we further hypothesized that ATP potentially neutralizes Ply already in the extracellular space. MST analyses revealed that, albeit weak, ATP and Ply interact with each other (Figure 6). ATP and even ADP bind to Ply in a mM-range (Figures 6A and 6C). In contrast, dATP did not show interactions with Ply (Figure 6B). These results are further supported by flow cytometry and FESEM analyses. Ply was readily detectable on about $50 \%$ 
of total neutrophil population post Ply stimulations (Figure 6D and Supplementary Figure S4). Addition of $3 \mathrm{mM}$ ATP to Ply stimulations almost completely abolished Ply binding to neutrophils (Figure 6D). FESEM analyses of neutrophil morphology confirmed their activation post sub-lytic Ply stimulations (Supplementary Figure S5A) which were abolished by addition of ATP. However, inter-individual variations were also detected (Supplementary Figure S5B). In contrast to donor 1, addition of ATP did not inhibited Ply-mediated neutrophil activation of donor 2 (Supplementary Figure S5). 


\section{DISCUSSION}

Neutrophils are the most abundant leukocytes in humans and the first recruited responders at the site of infection. In this study, we show that Ply is one of the major pneumococcal neutrophil activators. At sub-lytic concentrations, Ply induced degranulation, resulting in a release of resistin and other granule components. Notably, these actions are neutralized by extracellular ATP. Analyses of resistin and the whole proteome secreted by neutrophils revealed that addition of ATP significantly diminished Ply-mediated neutrophil degranulation. This reduction in neutrophil activation can be attributed to the interaction of Ply with ATP. The Ply-ATP interaction in the extracellular space abolished accumulation of Ply on neutrophil surfaces and subsequently reduced their activation.

Hyper-inflammatory conditions, such as bacterial infections, are associated with the release of nucleotides and in particular ATP. During apoptosis or necrosis, ATP is actively or passively released from dying cells and in certain conditions ATP is detected in mM ranges [1]. In the extracellular compartment, ATP functions as a signaling molecule through the activation of purinergic P2 receptors [34]. Beneficial roles for extracellular ATP including a function as a chemoattractant are reported [35]. However, high ATP levels are also associated with devastating conditions such as sepsis [36]. Two subclasses of P2 receptors exist, metabotropic P2Y and ionotropic P2X receptors [34]. P2X receptors, including $P 2 X_{1} R$, $P 2 X_{3} R$ and $P 2 X_{7} R$ have low affinity for ATP and are activated by ATP in mM range [37]. $\mathrm{P} 2 \mathrm{X}_{7} \mathrm{R}$, which is expressed on neutrophils and monocyte-derived cells, activates inflammasome in a pro-caspase 1 dependent manner [38]. Furthermore, it was shown that loss-of-function mutations in the $P 2 X_{7} R$ gene are associated with increased susceptibility of the human host to tuberculosis [39]. The role of P2 receptors in pneumococcal infections is not clear yet. However, it was shown that vital pneumococci can suppress ATP mediated responses of alveolar epithelial cells [40]. Furthermore, Domon and colleagues have demonstrated that Ply co-localizes with $\mathrm{P} 2 \mathrm{X}_{7} \mathrm{R}$ [23]. In contrast to the study by Domon et al. [23], our data do not indicate such interactions. Our experiments revealed that despite its 
cytolytic properties, Ply also serves as a potent trigger of neutrophil activation. The global secretome analyses and resistin determination of sub-lytic Ply stimulations show that almost equal numbers and quantities of proteins are released by neutrophils as compared to lytic stimulations. Furthermore, pharmacological inhibition of $\mathrm{P} 2 \mathrm{X}_{7} \mathrm{R}$ did not reduce Ply-mediated activation, indicating that other neutrophil surface molecules might serve as potential Ply receptors. However, supplementation of Ply stimulations with $\mathrm{P} 2 \mathrm{X}_{7} \mathrm{R}$ agonist ATP almost completely abolished neutrophil degranulation. Further analyses revealed that ATP interacts with Ply at $\mathrm{mM}$ range and subsequently reduces Ply binding to neutrophils and their activation. Therefore, our data indicate that Ply actions are neutralized through ATP in the extracellular space. However, inter-individual differences in Ply-mediated neutrophil activation post addition of ATP were detected. It should be noted that viable wild-type and $\Delta p l y$ pneumococci induced cytolysis and degranulation of neutrophils to the same extent. Neutrophil activation is a multifactorial process that involves several bacterial surface associated and secreted factors [12]. One potential pneumococcal secreted factor contributing to the initial neutrophil activation is $\mathrm{H}_{2} \mathrm{O}_{2}$. In contrast to $\mathrm{H}_{2} \mathrm{O}_{2}$, Ply is released through autolysis of pneumococci and worsens the already established infection through its activating and cytolytic activities. Assuming that ATP is already released in response to $\mathrm{H}_{2} \mathrm{O}_{2}$-mediated cytolytic injury, it might dampen further Ply-mediated tissue pathology. In addition to ATP, even ADP binds to Ply. Whether ADP might also act as a potential Plyneutralizing factor is not clear yet. However, ADP is a potent stimulator of P2Y receptors and the whole $\mathrm{P} 2$ receptor signaling is usually terminated by the conversion of ATP and ADP to AMP and adenosine by ectonucleotidases like CD39 or CD73 [41]. The balance between ATP/ADP and adenosine levels is crucial. Despite its beneficial actions, excessive release of ATP can contribute to the inflammatory tissue damage, while high levels of adenosine are mostly associated with immune suppression [36]. In line with this, Bou Ghanem and colleagues have shown that ATP-mediated neutrophil entry into the lungs correlates with initial control of pneumococcal burden [14]. However, as the infection progressed, further neutrophils influx was detrimental for bacterial clearance [14]. The authors linked the 
observed phenotype to the loss of CD73 expression [42]. Adenine production by CD73 was crucial to sustain antimicrobial efficacy of neutrophils [42]. However, the role of purinergic receptors and Ply was not explored in these studies.

Taken together, the data presented in this study support a concept that extracellular ATP has potentially a beneficial role in infections. It neutralizes excessive Ply-mediated neutrophil activation. Considering the great stimulatory capacity of pneumococci, it seems plausible that Ply-mediated neutrophil degranulation and activation may play a central role in tissue pathology in pneumococcal infection. On this note, whether such ATP actions apply only to Ply or also other cholesterol-dependent cytolysins, including streptolysin $\mathrm{O}$, listeriolysin $\mathrm{O}$ or suilysin and how such binding occurs biochemically warrant further studies. 


\section{Notes}

Acknowledgements. We would like to thank all volunteers for blood donation. Ulrike Lissner and Ina Schleicher are acknowledged for technical assistance.

Author contributions. F.C., B.K., and N.S. designed the study. F.C., B.K., M.G.S., S.S., S.B.S., G.B., T.T., M.R., and N.S. performed the experiments. F.C., B.K., M.G.S., S.M., M.R., and N.S. analyzed the data. F.C. and N.S. wrote the manuscript. F.C., B.K., M.G.S., S.S., S.B.S., G.B., S.M., T.T., M.R., S.H., U.V., and N.S. read, edited, and reviewed the manuscript.

Financial support. This research is supported by Federal Excellence Initiative of Mecklenburg Western Pomerania and European Social Fund (ESF) Grant Kolnfekt (ESF_14-BM-A55-0001_16; ESF_14-BM-A55-0003_16), Deutsche Forschungsgemeinschaft (DFG, 407176682), and The Swedish Society of Medicine (SLS-586271).

Potential conflicts of interest. All authors: No reported conflicts of interest. 


\section{References}

1. Idzko M, Ferrari D, Riegel AK, Eltzschig HK. Extracellular nucleotide and nucleoside signaling in vascular and blood disease. Blood 2014; 124:1029-37.

2. Ia Sala A, Ferrari D, Di Virgilio F, Idzko M, Norgauer J, Girolomoni G. Alerting and tuning the immune response by extracellular nucleotides. J Leukoc Biol 2003; 73:339-43.

3. Falzoni S, Donvito G, Di Virgilio F. Detecting adenosine triphosphate in the pericellular space. Interface Focus 2013; 3:20120101.

4. Trautmann A. Extracellular ATP in the immune system: more than just a "danger signal". Sci Signal 2009; 2:pe6.

5. Chen $Y$, Corriden $R$, Inoue $Y$, et al. ATP release guides neutrophil chemotaxis via P2Y2 and A3 receptors. Science 2006; 314:1792-5.

6. Geary C, Akinbi H, Korfhagen T, Fabre JE, Boucher R, Rice W. Increased susceptibility of purinergic receptor-deficient mice to lung infection with Pseudomonas aeruginosa. Am J Physiol-Lung C 2005; 289:L890-L5.

7. Brinkmann V, Reichard U, Goosmann C, et al. Neutrophil extracellular traps kill bacteria. Science 2004; 303:1532-5.

8. Kolaczkowska E, Kubes P. Neutrophil recruitment and function in health and inflammation. Nat Rev Immunol 2013; 13:159-75.

9. Lee WL, Harrison RE, Grinstein S. Phagocytosis by neutrophils. Microbes Infect 2003; 5:1299-306.

10. Borregaard N, Sorensen OE, Theilgaard-Monch K. Neutrophil granules: a library of innate immunity proteins. Trends Immunol 2007; 28:340-5.

11. Lacy P. Mechanisms of degranulation in neutrophils. Allergy Asthma Clin Immunol 2006; 2:98108.

12. Snall J, Linner A, Uhlmann J, et al. Differential neutrophil responses to bacterial stimuli: Streptococcal strains are potent inducers of heparin-binding protein and resistin-release. Sci Rep 2016; 6:21288.

13. UhImann J, Siemens N, Kai-Larsen Y, et al. Phosphoglycerate Kinase-A Novel Streptococcal Factor Involved in Neutrophil Activation and Degranulation. J Infect Dis 2016; 214:1876-83.

14. Bou Ghanem EN, Clark S, Roggensack SE, et al. Extracellular Adenosine Protects against Streptococcus pneumoniae Lung Infection by Regulating Pulmonary Neutrophil Recruitment. PLoS Pathog 2015; 11:e1005126.

15. Siemens N, Oehmcke-Hecht S, Mettenleiter TC, Kreikemeyer B, Valentin-Weigand $P$, Hammerschmidt S. Port d'Entree for Respiratory Infections - Does the Influenza A Virus Pave the Way for Bacteria? Front Microbiol 2017; 8:2602.

16. McCullers JA. Insights into the interaction between influenza virus and pneumococcus. Clin Microbiol Rev 2006; 19:571-+.

17. Valles J, Diaz E, Martin-Loeches I, et al. Evolution over a 15-year period of the clinical characteristics and outcomes of critically ill patients with severe community-acquired pneumonia. Med Intensiva 2016; 40:238-45.

18. Hammerschmidt S, Rohde M, Preissner KT. Extracellular Matrix Interactions with Gram-Positive Pathogens. Microbiol Spectr 2019; 7.

19. Mitchell TJ, Dalziel CE. The biology of pneumolysin. Subcell Biochem 2014; 80:145-60.

20. Kadioglu A, Taylor S, lannelli F, Pozzi G, Mitchell TJ, Andrew PW. Upper and lower respiratory tract infection by Streptococcus pneumoniae is affected by pneumolysin deficiency and differences in capsule type. Infect Immun 2002; 70:2886-90.

21. Vogele $M$, Bhaskara RM, Mulvihill E, et al. Membrane perforation by the pore-forming toxin pneumolysin. P Natl Acad Sci USA 2019; 116:13352-7.

22. Subramanian K, Neill DR, Malak HA, et al. Pneumolysin binds to the mannose receptor $C$ type 1 (MRC-1) leading to anti-inflammatory responses and enhanced pneumococcal survival. Nat Microbiol 2019; 4:62-70. 
23. Domon H, Oda M, Maekawa T, Nagai K, Takeda W, Terao Y. Streptococcus pneumoniae disrupts pulmonary immune defence via elastase release following pneumolysin-dependent neutrophil lysis. Sci Rep-Uk 2016; 6.

24. Nel JG, Durandt C, Theron AJ, et al. Pneumolysin mediates heterotypic aggregation of neutrophils and platelets in vitro. J Infection 2017; 74:599-608.

25. Ullah I, Ritchie ND, Evans TJ. The interrelationship between phagocytosis, autophagy and formation of neutrophil extracellular traps following infection of human neutrophils by Streptococcus pneumoniae. Innate Immun-London 2017; 23:413-23.

26. Siemens N, Fiedler T, Normann J, et al. Effects of the ERES pathogenicity region regulator Ralp3 on Streptococcus pyogenes serotype M49 virulence factor expression. J Bacteriol 2012; 194:3618-26. 27. Siemens N, Chakrakodi B, Shambat SM, et al. Biofilm in group A streptococcal necrotizing soft tissue infections. Jci Insight 2016; 1:e87882.

28. Wienken CJ, Baaske P, Rothbauer U, Braun D, Duhr S. Protein-binding assays in biological liquids using microscale thermophoresis. Nat Commun 2010; 1:100.

29. Uhlmann J, Rohde M, Siemens N, et al. LL-37 Triggers Formation of Streptococcus pyogenes Extracellular Vesicle-Like Structures with Immune Stimulatory Properties. J Innate Immun 2016; 8:243-57.

30. Blankenburg S, Hentschker C, Nagel A, et al. Improving Proteome Coverage for Small Sample Amounts: An Advanced Method for Proteomics Approaches with Low Bacterial Cell Numbers. Proteomics 2019; 19:e1900192.

31. Suomi T, Elo LL. Enhanced differential expression statistics for data-independent acquisition proteomics. Sci Rep 2017; 7:5869.

32. Suomi T, Seyednasrollah F, Jaakkola MK, Faux T, Elo LL. ROTS: An R package for reproducibilityoptimized statistical testing. PLoS Comput Biol 2017; 13:e1005562.

33. Raudvere U, Kolberg L, Kuzmin I, et al. g:Profiler: a web server for functional enrichment analysis and conversions of gene lists (2019 update). Nucleic Acids Res 2019; 47:W191-W8.

34. Khakh BS, Burnstock G. The Double Life of Atp. Sci Am 2009; 301:84-+.

35. Mihm S. Danger-Associated Molecular Patterns (DAMPs): Molecular Triggers for Sterile Inflammation in the Liver. Int J Mol Sci 2018; 19.

36. Ledderose $\mathrm{C}$, Bao $\mathrm{Y}$, Kondo $\mathrm{Y}$, et al. Purinergic Signaling and the Immune Response in Sepsis: A Review. Clin Ther 2016; 38:1054-65.

37. Karmakar M, Katsnelson MA, Dubyak GR, Pearlman E. Neutrophil P2X7 receptors mediate NLRP3 inflammasome-dependent IL-1 beta secretion in response to ATP. Nat Commun 2016; 7:10555.

38. Surprenant A, North RA. Signaling at purinergic P2X receptors. Annu Rev Physiol 2009; 71:333-59. 39. Coutinho-Silva R, Ojcius DM. Role of extracellular nucleotides in the immune response against intracellular bacteria and protozoan parasites. Microbes Infect 2012; 14:1271-7.

40. Olotu C, Lehmensiek F, Koch B, et al. Streptococcus pneumoniae inhibits purinergic signaling and promotes purinergic receptor P2Y2 internalization in alveolar epithelial cells. J Biol Chem 2019; 294:12795-806.

41. Yegutkin GG. Nucleotide- and nucleoside-converting ectoenzymes: Important modulators of purinergic signalling cascade. Bba-Mol Cell Res 2008; 1783:673-94.

42. Siwapornchai N, Lee JN, Tchalla EYI, et al. Extracellular adenosine enhances the ability of PMNs to kill Streptococcus pneumoniae by inhibiting IL-10 production. J Leukoc Biol 2020. 


\section{Fugure Legends}

Figure 1. S. pneumoniae is efficiently killed by cellular blood components. TIGR4 wt or $\Delta p l y$ mutant were incubated in THY $(A)$, human plasma $(B)$, or human blood $(C)$ for $2 \mathrm{~h}$ and bacterial numbers were determined. Percentage of initial inoculum is shown. $D$, Resistin release by the whole human blood in response to bacterial infection. The data show results from four independent experiments using four donors $(n=4)$. Horizontal lines depict median values. The level of significance was determined using two-tailed Mann-Whitney $U$ test (n.s., not significant).

Figure 2. Pneumolysin is a cytoplasmic pneumococcal trigger of neutrophil degranulation. Purified neutrophils were stimulated with bacteria $(A$ and $B)$ or with cytoplasmic fraction of pneumococcal lysates at indicated dilutions $(C$ and $D)$ for $2 \mathrm{~h}$ and cytotoxicity $(A$ and $C)$ and neutrophil degranulation ( $B$ and $D$ ) were assessed. Each symbol represents purified neutrophils from one donor $(n=4)$. Horizontal lines depict median values. The level of significance between the groups was determined using Kruskal Wallis test with Dunn's posttest (n.s., not significant; ${ }^{\star} p<0.05 ;{ }^{* *} p<0.01$ ).

Figure 3. Pneumolysin activates human neutrophils. Purified primary neutrophils from healthy volunteers were stimulated with indicated concentrations $\left[\mu \mathrm{g} \mathrm{ml}^{-1}\right]$ of pneumolysin for $2 \mathrm{~h}$ and cytotoxicity $(A)$ and neutrophil degranulation $(B)$ were assessed (autol., autologous; w/o, without). Each symbol represents purified neutrophils from one donor ( $n \geq 4)$. Horizontal lines depict median values. The level of significance between the groups was determined using Kruskal Wallis test with Dunn's post-test (n.s., not significant; ** $p<0.01)$. (C) Representative immuno-fluorescence micrographs of pneumolysin (Ply) on primary neutrophils after $2 \mathrm{~h}$ of stimulation. 
Figure 4. Extracellular ATP neutralizes pneumolysin-dependent neutrophil activation. $(A)$ Purified primary neutrophils from healthy volunteers were stimulated with indicated concentrations of single agents or in combination for $2 \mathrm{~h}$ and neutrophil degranulation was assessed. Each symbol represents purified neutrophils from one donor $(n=10)$. Horizontal lines depict median values. $(B)$ Total numbers of secreted proteins identified in neutrophil supernatants post indicated stimulations $(n=5)$. The level of significance between the groups was determined using Kruskal Wallis test with Dunn's post-test (n.s., not significant; * $\left.p<0.05 ;{ }^{* *} p<0.01 ;{ }^{* *} p<0.001\right) .(C)$ Principal component analysis of secreted proteins. Each dot represents one donor $(n=5)$. The ellipses indicate the calculated $95 \%$ probability region for a bivariate normal distribution with an average center of groups. (D) Heat map of significantly different protein abundancies in neutrophil secretome post $0.3125 \mu \mathrm{g}$ vs. $2.5 \mu \mathrm{g}$ Ply stimulations. The data are displayed as signal $\log _{2}$ ratios from five donors $(n=5)$.

Figure 5. Comparison of neutrophil secretome profiles in response to Ply and Ply-ATP stimulations. ( $A$ ) Heat map of significantly different protein abundances $(q<0.05 ; \mathrm{FC}>2)$ in neutrophil secretome post $0.3125 \mu \mathrm{g} / 3 \mathrm{mM}$ ATP vs. $0.3125 \mu \mathrm{g}$ and $2.5 \mu \mathrm{g}$ Ply/3 mM ATP vs. $2.5 \mu \mathrm{g}$ Ply stimulations. The data are displayed as signal $\log _{2}$ ratios from five donors $(n=5)$. (B) Heat map highlighting significant differences of protein/peptide abundancies in neutrophils secretomes from $(A)$. The data are displayed as mean protein intensities of the indicated stimulations from five donors $(n=5)$. (C) Top five down-regulated pathways by addition of ATP to pneumolysin stimulations. Displayed are adjusted $p$-values as determined by functional profiling in the $g:$ Profiler database (version e98_eg45_p14_ce5b097). 
Figure 6. ATP and Ply interact with each other. Purified recombinant Ply was NT-647 labeled and binding affinities with $\operatorname{ATP}(A), \operatorname{dATP}(B)$ and $\operatorname{ADP}(C)$ were determined using microscale thermophoresis. Each symbol represents mean values $\pm S D$ from three independent experiments $(n=3)$. The $K_{d}$ values are indicated in the corresponding figures. (D) Summary of the FACS data of Ply-positive neutrophils post indicated stimulations. Each symbol represents purified neutrophils from one donor $(n=4)$. Horizontal lines depict median values. 
Figure 1

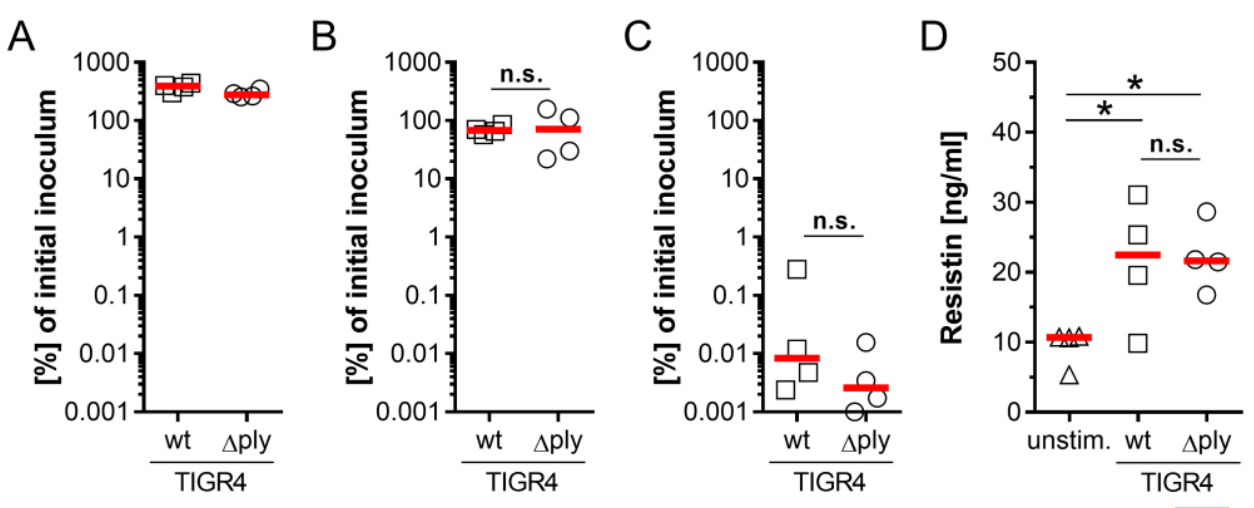


Figure 2
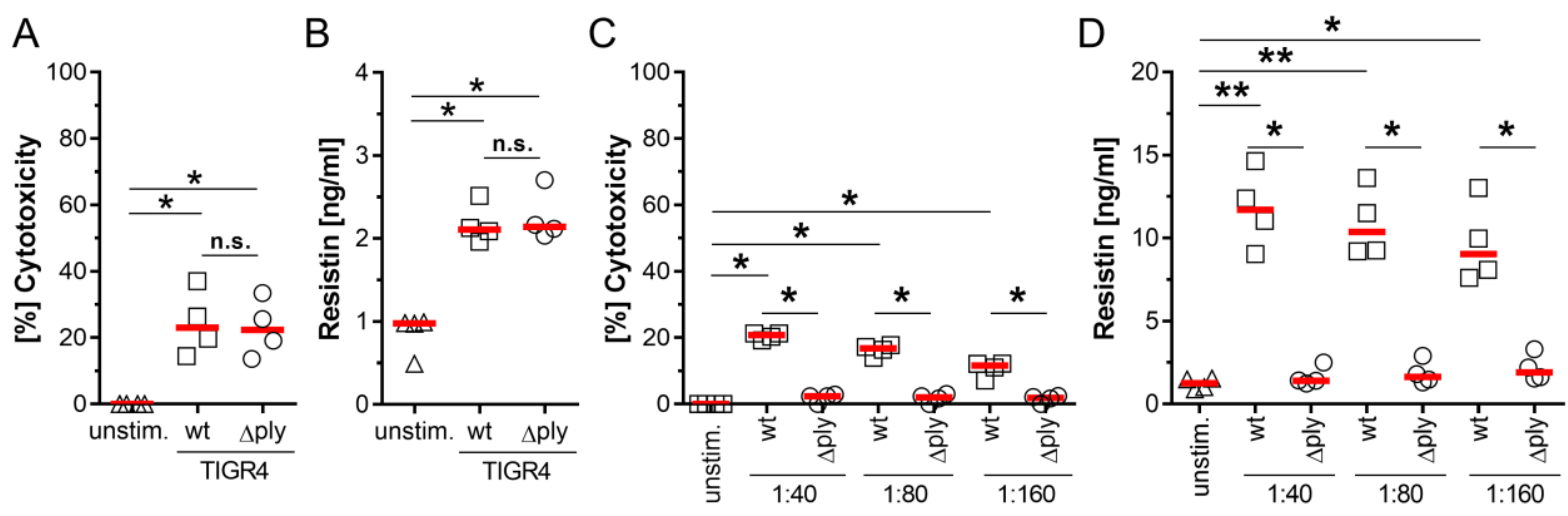
Figure 3
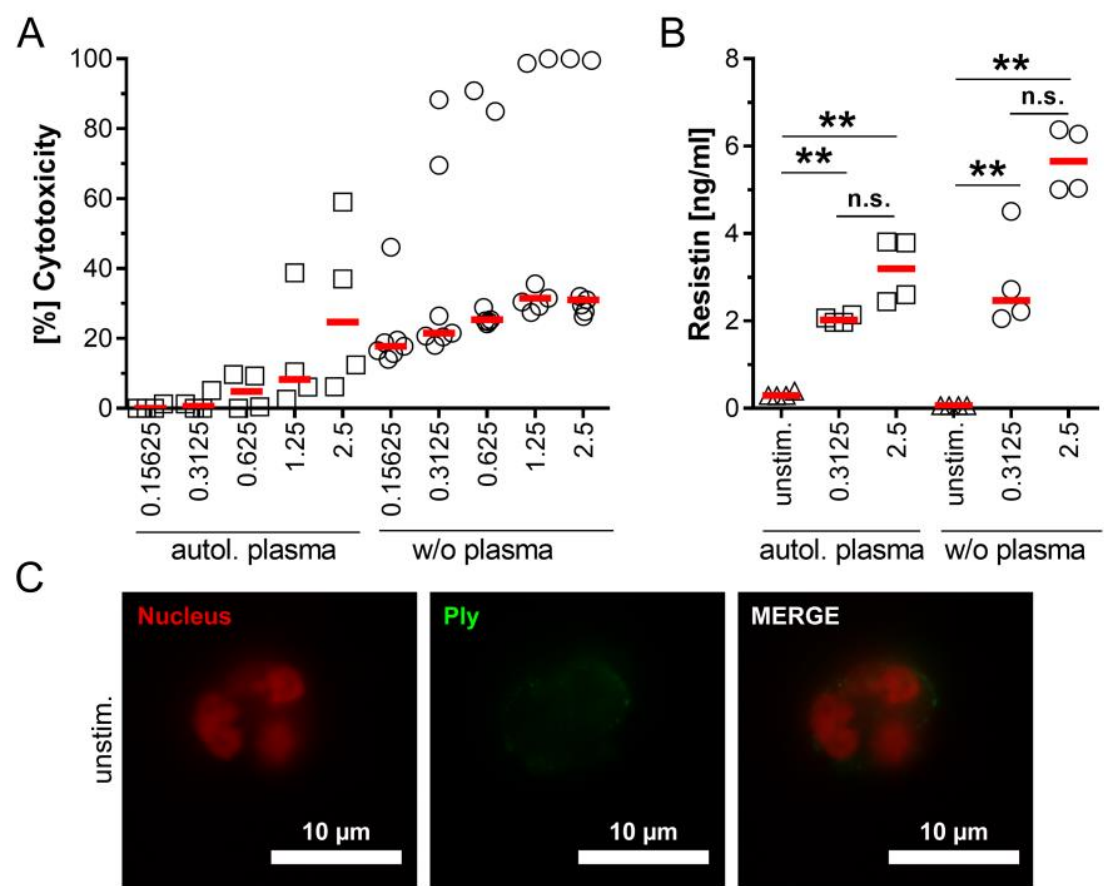

autol. plasma $\overline{\text { w/o plasma }}$
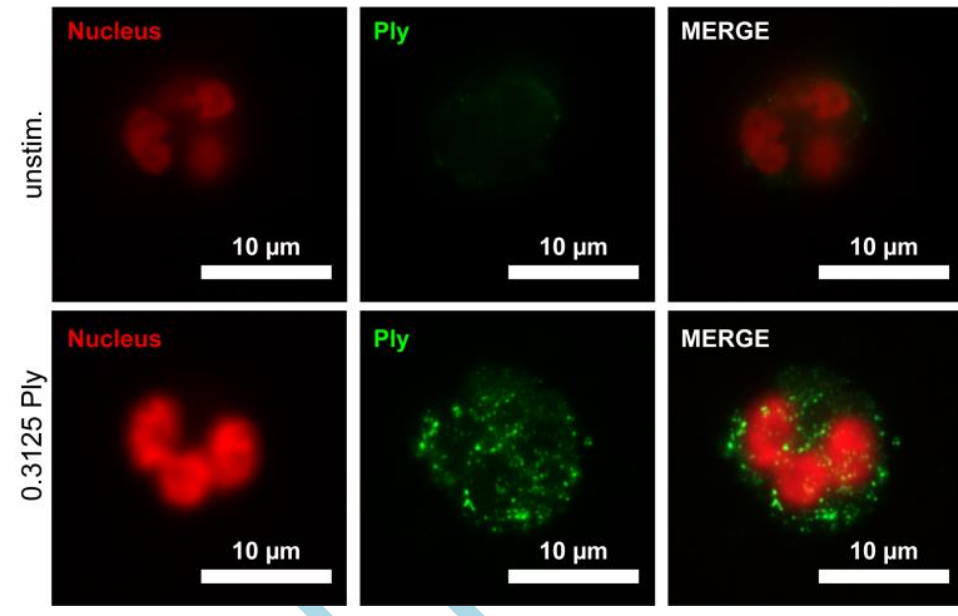
Figure 4
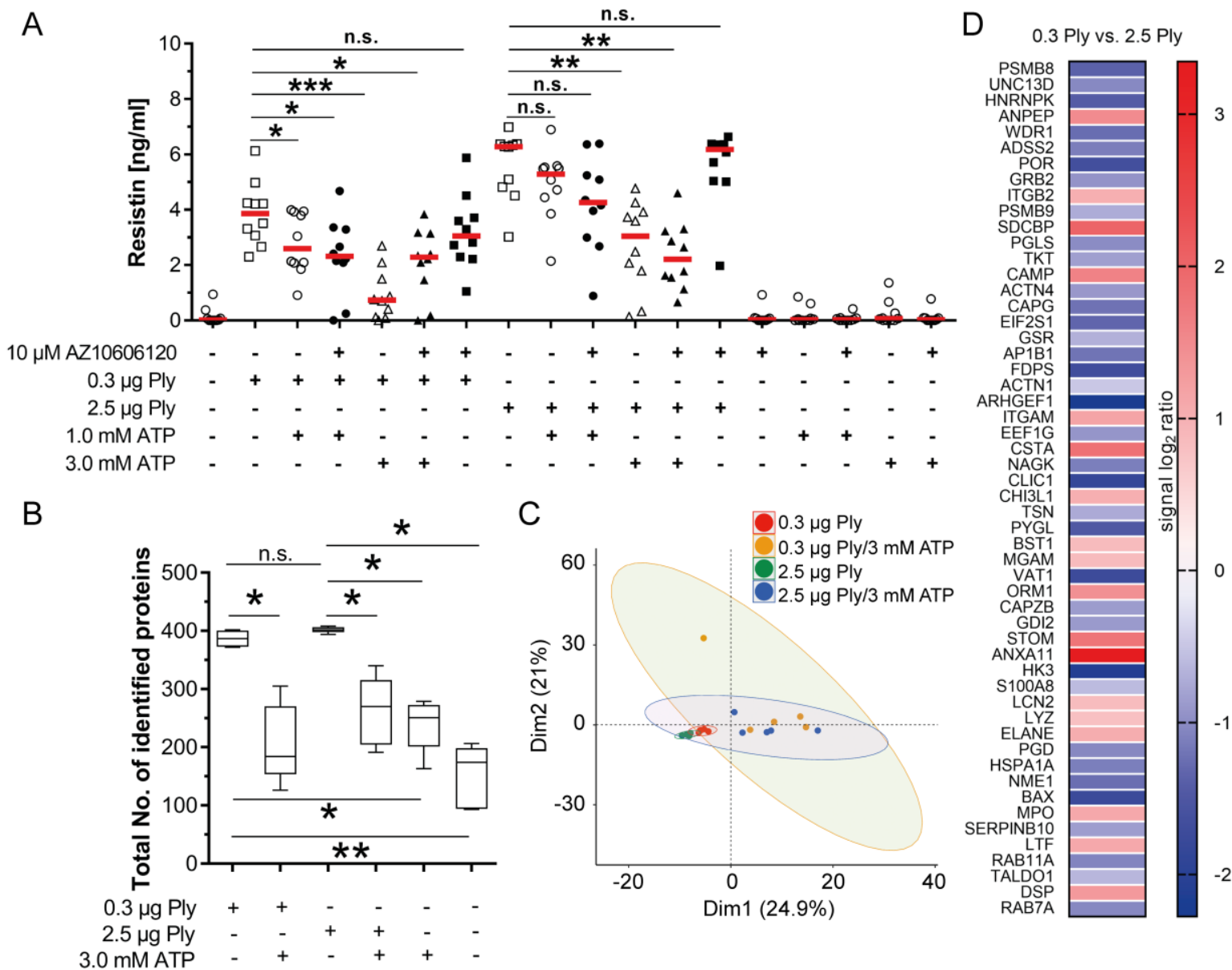
Figure 5

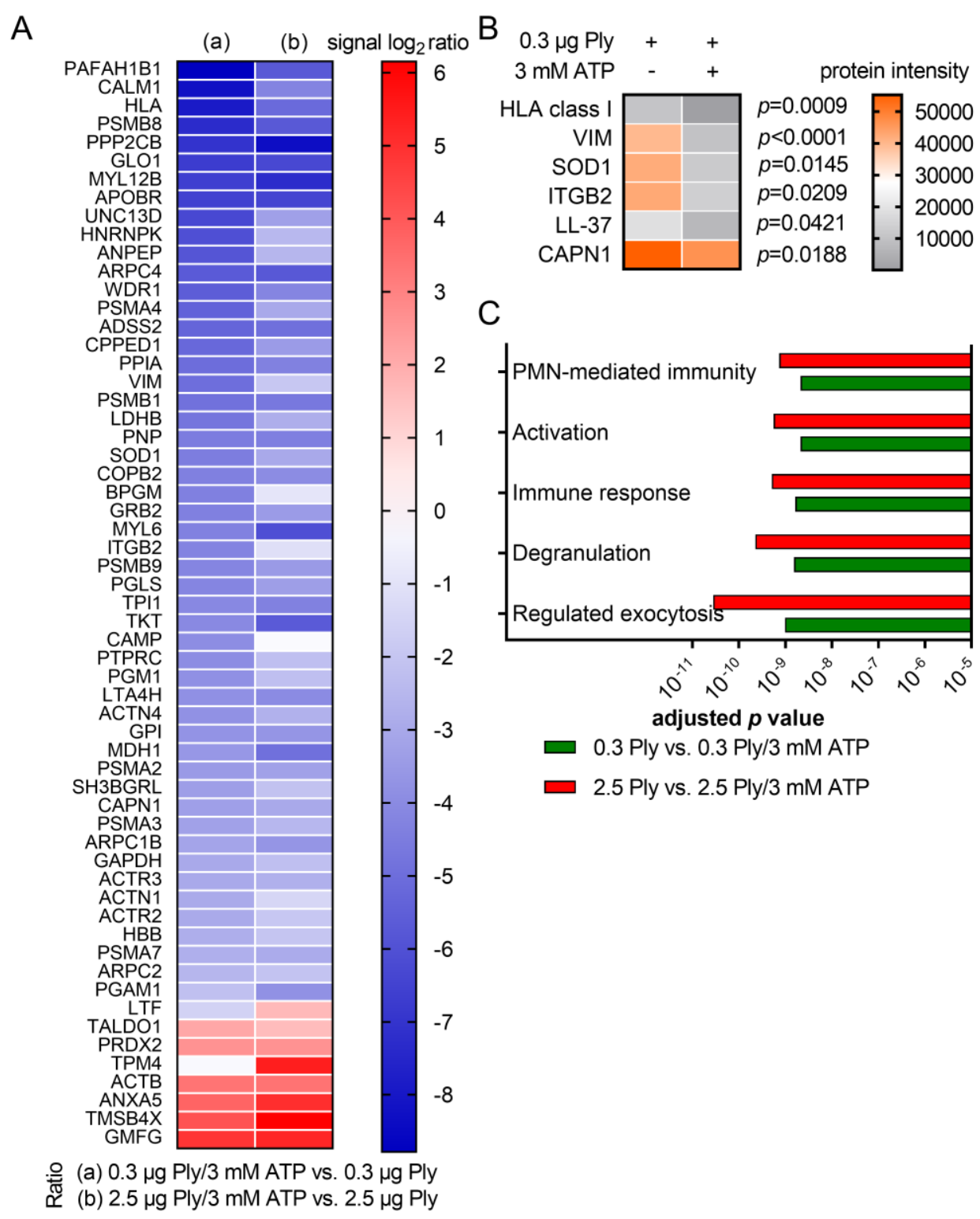


Figure 6
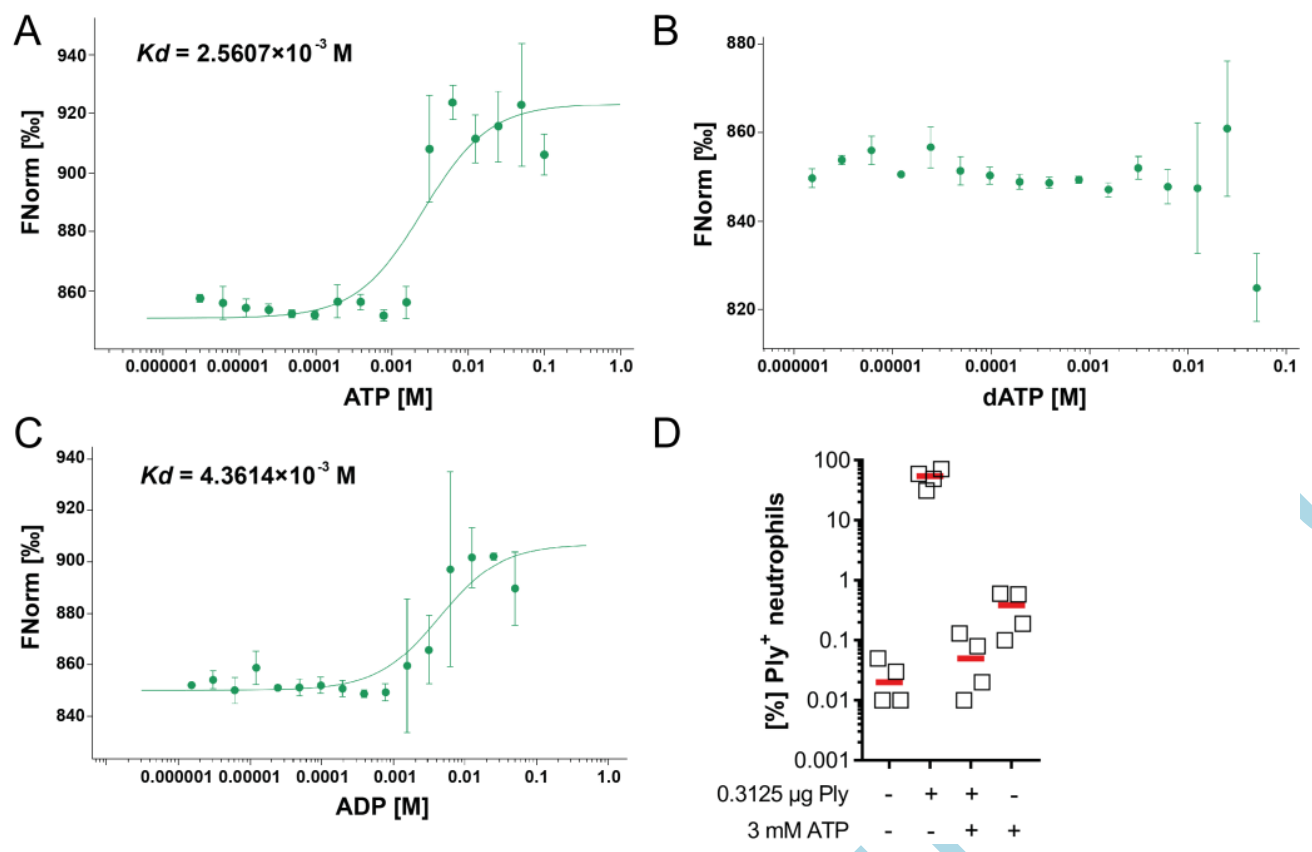